2. Das Verfahren ist außerordentlich spezifisch, da die Intensität der charakteristischen Phosphor-Atomlinie ausgenutzt wird und es wegen der hohen Temperatur völlig gleichgültig ist, in welcher Bindung und in welcher Mischung mit anderen Elementen es vorliegt.

3. Die Probenvorbereitung und Messung sind einfach und benötigen wenig Zeit. Das Ergebnis steht spätestens nach 30 Minuten zur Verfügung. Bei der chemischen Methode werden allein für die Veraschung 5 Stunden benötigt.

Ein wesentlicher Nachteil ist es, daß der benutzte Plasmabogen noch nicht im Handel ist.

Frau I. Hormerster und Frau R. Ristig danken wir für die sorgfältige Durchführung der chemischen Bestimmungen.

\title{
Literatur
}

1. Herrmann, R. und K. Rötger, diese Z. 4, 217 (1966). - 2. Zöllner, N., diese Z. 1, 18 (1963). - 3. Bartlett, G. R., J. biol. Chemistry 234, 466 (1959). - 4. BürTNER, H., diese Z. 3, 69 (1965).
- 5. Gebeitein, H. und I. Herte, Statistische Urteilsbildung, Springer-Verlag, Berlin-Göttingen-Heidelberg (1951). 6. Rausch, L. und H. GrauL, Ärztl. Wschr. 4, 591. (1949).
Dr. med. Dr. rer. nat. D. Stamm

Klin.-chem. Abt. an der Chirurg. Universitätsklinik

63 Gießen, Klinikstr. 37

\section{Mikrolitermethode zur Bestimmung von Serum-Eisen}

\author{
Von D. Stamm, HJ. Staudinger und W. Weis \\ Aus dem Physiologisch-chemischen Institut (Direktor: Prof. Dr. Hj. Staudinger) und der Klinisch-chemischen Abteilung \\ (Leiter: Dr. Dr. D. Stam m) an der Chirurgischen Universitätsklinik der Universität Gießen
}

(Eingegangen am 6. April 1966)

\begin{abstract}
Es wird eine Mikrolitermethode zur Bestimmung des Serumeisens unter Verwendung eines neuen Komplexbildners mit hohem molaren Extinktionskoeffizient beschrieben, für die nur $50 \mu l$ Serum benötigt werden und die zuverlässiger als die Millilitermethode ist, wie sich aus der Qualitätskontrolle und Richtigkeitsprüfung ergibt.

A microlitre method for the determination of serum iron is described. A new complexing agent with a high molar extinction coefficient is used. Only $50 \mu l$ of serum are required and, as shown by quality control and accuracy tests, it is more reliable than the millilitre method.
\end{abstract}

Bei den bisher üblichen Milliliternethoden zur Bestimmung des Serum-Eisens gibt es drei Schwierigkeiten: Erstens die Beschaffung der einwandfreien Probemengen von 1-2 Milliliter; zweitens die genaue Einhaltung des $\mathrm{pH}$ im Bestimmungsansatz; drittens die Reinigung der Glasgeräte von Eisenspuren (1). Nachdem nun KRÖHNKE und STAUDINGER mit ihren Mitarbeitern (2) einen neuen Komplexbildner mit einem ungewöhnlich hohen, nur wenig vom $\mathrm{pH}$ abhängigen molaren Extinktionskoeffizienten $(\varepsilon=26900)$ für die Serumeisenbestimmung eingeführt haben, erscheint es sinnvoll, dieses Verfahren als Mikrolitermethode (3) auszuarbeiten und seine Zuverlässigkeit, Präzision und Richtigkeit zu prüfen. Denn zu allen anderen Vorzügen der Mikrolitermethoden kommt der Vorteil, daß man bei dem Mikrolitersystem „Eppendorf $^{\text {('1 }}$ ) die Reaktionsgefäße und die Pipettenspitzen unmittelbar aus den zugeschweißten Beuteln des Herstellers entnehmen und ohne vorherige Reinigung für die Eisenbestimmung verwenden kann (4). Da man nur ganz geringe Reagenzienmengen benötigt, kann man ,super- * reine" Chemikalien verwenden; dadurch wird eine Vielzahl von Fehlermöglichkeiten ausgeschaltet.

\section{Methode}

Die Untersuchungen werden mit dem Mikrolitersystem „Eppendorf“ durchgeführt. Die Konzentration der

1) Hersteller: Fa. „Eppendorf Gerätebau“, Netheler und Hinz GmbH, Hamburg.
Reagenzien und der Volumina sind auf dieses System abgestimmt.

\section{Reagenzien}

Für die Lösungen und die Reaktionsansätze wird eisenfreies aus Quarz destilliertes, entmineralisiertes Wasser verwendet.

$6 \mathrm{~N}$-Salzsäure: Aus $60 \mathrm{ml}$ Salzsäure $(\mathrm{D}=1,15)$,ultrarein“ (Fa. Merck Nr. 318), die etwa $9,5 \mathrm{~N}$ ist, werden $95 \mathrm{ml} 6 \mathrm{~N}$ Salzsäure durch Zugabe von $35 \mathrm{~m} /$ Wasser hergestellt.

20proz. Tricbloressigsäure: $\mathrm{Zu} 30 \mathrm{ml} 40$-proz. Trichloressigsäure $\left(\mathrm{CCl}_{3} \mathrm{COOH}\right)$ p. a. zur Eisenbestimmung (Fa. Merck Nr. 811) sind $30 \mathrm{~m} l$ Wasser hinzuzugeben.

3,5 M Natriumacetat-Lösung pH 4,8-5,4: Man löst 28,7 g Natriumacetat $\left(\mathrm{CH}_{3} \mathrm{COONa}\right.$ ) „ultrarein“ (Fa. Merck Nr. 6264) in $100 \mathrm{ml}$ Wasser.

Ascorbinsäure $1,5 \cdot 10^{-2} \mathrm{M}:$ Man löst $2,64 \mathrm{mg}$ p. a. Ascorbinsäure (Fa. Merck Nr. 127) in $100 \mathrm{ml}$ Wasser.

Eisenreagenz: 2,6-Di-[pyridyl-(2)]-4-[p-methoxy-phenyl]-pyridin $5 \cdot 10^{-4}$ M. Man löst $17,0 \mathrm{mg}$ des Reagenzes, dessen Darstellung bei KRöHNKe und StaUdinger (2) beschrieben ist, in $100 \mathrm{ml}$ Isopropylalkohol (Fa. Merck Nr. 9634).

Eisenstandardlösung $1000 \mu \mathrm{g} \mathrm{Fe} / 100 \mathrm{ml}$ : Man wiegt $70,4 \mathrm{mg} \mathrm{Mobr}-$ sches Salz, Ammoniumeisen-(II)-sulfat, $\left[\left(\mathrm{NH}_{4}\right)_{2} \mathrm{Fe}\left(\mathrm{SO}_{4}\right)_{2} \cdot 6 \mathrm{H}_{2} \mathrm{O}\right.$, $\mathrm{M}=392,16]$ p. a. (Fa. Merck Nr. 3792) ein und füllt in einem Meßkolben auf $1000 \mathrm{~m} l$ auf.

Arbeitsstandard $100 \mu \mathrm{g} / 100 \mathrm{ml}$ : Die Eisenstandardlösung wird in einem $100-\mathrm{m} /$-Meßkolben $1+9$ mit Wasser verdünnt. 


\section{Arbcitsgang}

Man setzt in jeder Serie 3 Leerwerte, 3 Standards und je 1 Probe an und verfährt weitcr nach Tabelle 1 :

Tab. 1

Pipcttierschema (Mengen in $\mu l$ )

\begin{tabular}{lccc}
\hline Reagenz & Leerwert & Standard & Probe \\
\hline Wasser & 50 & - & - \\
Standard & - & 50 & - \\
Serum & - & - & 50 \\
$6 \mathrm{~N} \mathrm{HCl}$ & 50 & 50 & 50
\end{tabular}

Die Ansätze auf dem Rüttler gut mischen und $15 \mathrm{Min}$. stehen lassen

$\begin{array}{llll}\text { Wasser } & 100 & . .100 & 100 \\ \text { Trichloressigsäure } & 100 & 100 & 100\end{array}$

Die Ansätze auf dem Rüttler gut mischen, für 3 Min. in der Mikrozentrifuge zentrifugieren; Uberstand abnehmen in neues

$\begin{array}{lrrr}\text { Rea.ktionsgefäß } & 200 & 200 & 200 \\ \text { Natriumacetat } & 200 & 200 & 200 \\ \text { Ascorbinsäure } & 50 & 50 & 50 \\ \text { Eisen-Reagenz } & 500 & 500 & 500\end{array}$

Die Ansätze auf dem Rüttler gut mischen und 15 Min. stehen lassen

Messung

Im Spektralphotometer (Fa. Zciss „PMQ I“ mit Mikroansatz) werden bei $568,5 \mathrm{~m} \mu$ in Lowry-Bessy Küvetten von $2 \mathrm{~cm}$ Schichtdicke die Extinktionen [E] der Standards und der Proben gegen Wasser abgelesen. Den Leerwert prüft man vorher durch Ablesen seiner Extinktion gegen Wasser.

Berechnumg

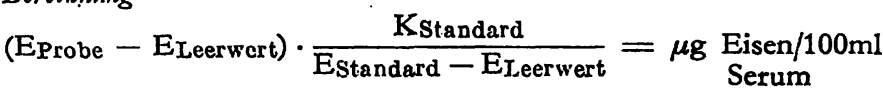

$\mathrm{K}=$ Konzentration

Eichung

Man gibt von der Eisenstandardlösung $(1000 \mu \mathrm{g} / 100 \mathrm{~m} l)$ die folgenden Mengen mit geeichten Vollpipetten in $100 \mathrm{ml}$ Meßkolben und füllt mit Wasser bis zur Marke auf:

Tab. 2

Pipettierschema für die Eichkurvenverdünnung

\begin{tabular}{lrrrrrrrr}
\hline Staridard & & & & & & & & \\
$1000 \mu \mathrm{g} / 100 \mathrm{~m} l(\mathrm{~m} l)$ & 4 & 6 & 8 & 10 & 15 & 20 & 30 & 40 \\
Endvolumen $(\mathrm{m} l)$ & 100 & 100 & 100 & 100 & 100 & 100 & 100 & 100 \\
$\begin{array}{l}\text { Konzentration } \\
\quad(\mu \mathrm{g} / 100 \mathrm{~m} l)\end{array}$ & 40 & 60 & 80 & 100 & 150 & 200 & 300 & 400 \\
\hline
\end{tabular}

Mit jeder Verdünnung werden 3 Ansätze nach dem Pipettierschema der Tabelle 1 gemacht und die abgelesenen Extinktionen nach $\mathrm{Ab}-$ zug des Leerwertes gemittelt. Die Extinktionen sind aus der Eichkurve (Abb. 1) abzulesen. Die Eichkurve ist bis zu $400 \mu \mathrm{g} / 100 \mathrm{~m} l$ völlig linear. Der Durchmesser der Punkte beträgt 3 Standardabweichungen der Methode.

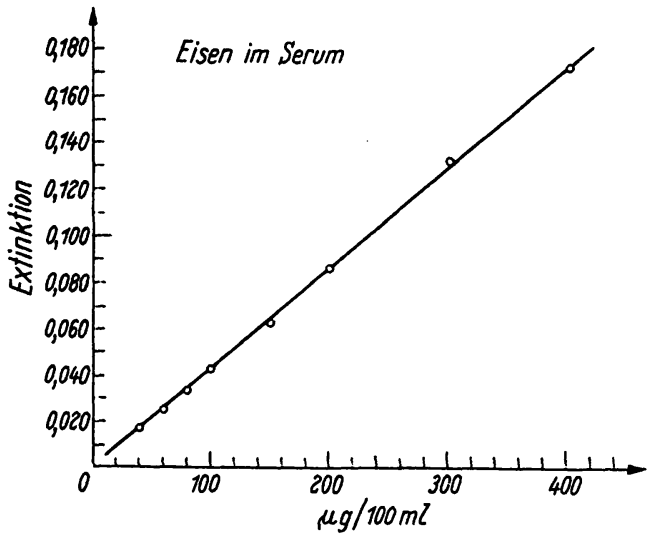

Abb. 1

Eichkurve zur Eisenbestimmung

\section{Kontrolle der Methode}

Die Präzision $(5,6)$ (precision) in der Serie wurde durch jeweils 25 Bestimmungen eines Serums hoher Eisenkonzentration und eines Serums niederer Eisenkonzentration geprüft. Die absoluten Werte der Standardabweichung sind in beiden Konzentrationsbereichen gleich, die Variationskoeffizienten entsprechend verschieden.

Die Präzision von Tag zu Tag ist aus einer Qualitätskontrolle (6) mit „Qualtrol“" ) über einen Monat errechnet. Im Verlaufe dieses Monats haben sich keine nennenswerten Abweichungen über den KontrollbereichMittelwert \pm 2 Standardabweichungen, hinaus ergeben, d. h. die Methode ist nie aus der Kontrolle geraten (Tab.3).

Die Richtigkeit $(5,6)$ (accuracy) wurde einmal durch eine tägliche Bestimmung von „Monitrol I“2) über 17 Tage geprüft; es exgab sich infolge der Mitführung eines inneren Standards keine wesentlich größere Abweichung als in der Serie. Der Variationskoeffizient betrug bei einem Mittelwert von $96 \mu \mathrm{g} / 100 \mathrm{ml} 1,68 \%$.

Zum anderen prüften wir die Richtigkeit durch $\mathrm{Zu}-$ mischversuche zu jeweils $1000 \mu l$ Qualtrol, dem wir zwischen 10 und $100 \mu l$ Eisenstammlösung zusetzten (Tab. 4):

Tab. 4

Wiederfindung

\begin{tabular}{lrrrr}
$\begin{array}{lrrrr}\text { Vorgegebene Kon- } \\
\text { zentration }(\mu \mathrm{g} / 100 \mathrm{~m} l)\end{array}$ & 187,3 & 147,8 & 123,6 & $109,5$. \\
$\begin{array}{l}\text { bestimmte Konzen- } \\
\text { tration }(\mu \mathrm{g} / 100 \mathrm{~m} l)\end{array}$ & 184,8 & 148,5 & 120,5 & 106,1 \\
Wiederfindung $(\%)$ & 98,7 & 100,5 & 97,5 & 96,9 \\
\hline
\end{tabular}

Tab. 3

Präzision der Eisenbestimmung

\begin{tabular}{lccc}
\hline & Mittelwert & Standardabweichung & Variationskoeffizient \\
$(\mu \mathrm{g} / 100 \mathrm{~m} l)$ & $(\mu \mathrm{g} / 100 \mathrm{~m} l)$ & $\pm 1,63$ & 0,96 \\
In der Serie & 174,6 & $\pm 1,60$ & 1,63 \\
unter Routinebedingungen & 95,4 & $\pm 1,62$ & 1,68 \\
von Tag zu Tag (Routinebedingungen) & 96,0 & & \\
\hline
\end{tabular}

2) Hersteller: Fa. Dade Reagents, Inc., Miami, USA; Alleinvettrieb für Deutschland: Asid-Institut GmbH. München 13, Schließfach 420. 


\section{Diskussion}

Die Mikrolitermethode hat gegenüber der Millilitermethode folgende Vorzüge:

1. Sie ist zuverlässiger; die Standardabweichung beträgt 1,6 gegenüber $2,7 \mu \mathrm{g} / 100 \mathrm{~m} l$ bei der Millilitermethode.

2. Die Probemenge beträgt ein Zehntel der Millilitermethode.

3. Sie ist billiger, weil die hohen Reinigungskosten für die Glasgeräte entfallen; man kann fabrikneue Kunststoff-Reaktionsgefäße und Pipettenspitzen verwenden.

4. Die Infektionsgefährdung ist wesentlich geringer als bei der Millilitermethode, weil die Versuchung, Proben mit dem Mund in die Pipetten anzusaugen, entfällt und die infizierten Reaktionsgefäße und Pipettenspitzen sofort weggeworfen werden.

Frau I. Hofmerster danken wir für die sorgfältige Durchführung der Bestimmungen.

\title{
Literatur
}

1. Govaniello, T. J. und T. Peters in: D. Seligson, Standard Methods of Clinical Chemistry, Bd. IV, S. 139-150, Academic Press, New York-London (1963). - 2. DegGaU, E., F. KröhNKE, K. E. Schmalke, HJ. Staudinger und W. Weis, diese Z. 3, 102 (1965). - 3. Bütrner, H., Dtsch. med. Wschr. 88, 910 (1963). 4. Gladtke, E., H. Bütrner und D. Stama, diese Z. 3, 61 (1965).
-5. BütTNER, H., Mikroliteranalysen, in: Handbuch dex Kinderheilkunde Bd. 2, 1. Teil, S. 860-874, Springer-Verlag, BerlinHeidelberg-New York (1966). 6. HenRY, R. I. und R. L. DRYer in: D. Seligson, Standard Methods of Clinical Chemistry Bd. IV, S. 205-237, Academic Press, New York-London (1963).

Dr. med. Dr. rer. nat. D. Stamm Klin.-chem. Abteilung an der Chirurg. Universitätsklinik

63 Gießen, Klinikstr. 37

\section{Die enzymatische Blutzucker-Bestimmung in vitro und in vivo mit dem Auto-Analyzer}

\author{
Von E. KaWERAU \\ Aus dem Department of Chemical Pathalogy, St. James' Hospital, London
}

(Eingegangen am 28. Februar 1966)

\begin{abstract}
Im ersten Abschnitt wird die Bestimmung von Glukose in Blut- und Liquorproben mit dem Glukose-OxydasePeroxydase System beschrieben; o-Tolidin dient als Farbreagenz. Die Technik und die Grundprinzipien, die der Anlage des Schlauch-Verteilersystems zugrunde liegen, werden eingehend besprochen. Die Methode erlaubt es, 40 Bestimmungen in det Std. zu machen; von der Blutentnahme bis zum ausgewerteten Resultat werden nur 8 Min. benötigt. Im zweiten Abschnitt wird die Glukose im Blut laufend am Patienten bestimmt, der durch einen intravenösen Katheter direkt mit dem ,,Technicon“ Auto-Analyzer verbunden ist. Die Genauigkeit und Reproduzierbarkeit beider Methoden sind dargestellt und statistisch gesichert.

A rapid method is described for the determination of blood and c. s. f. glucose with the glucose-oxydase-peroxidase system employing o-tolidine as the colour reagent. Some of the fundamental principles underlying the construction of the manifold are discussed. The method allows 40 determinations per hour to be made and no more than 8 minutes need elapse after bleeding the patient and the readout of the result. In the in vivo technique, the patient is connected directly to the ,"Technicon" Auto-Analyzer by indwelling catheter in an ante-cubital vein, but basically, it is the same method. The accuracy and reproducibility of the method has been tested and the results have been submitted to the usual statistical check.
\end{abstract}

Eine schnelle, spezifische und störungsfreie Bestimmung der Glukose im Blut ist für die Klinik von größter Bedeutung. Unsere Methode, die in den letzten Jahren entwickelt wurde, hat sich als einfach und zuverlässig erwiesen, und da sie anderen Methoden gegenüber gewisse Vorzüge hat und vor allen Dingen schneller arbeitet, soll sie hier eingehend beschrieben werden. Außerdem soll. die Methode mir hier als Modell zur Erläuterung einiger Grundprinzipien der Konstruktion neuer VerteilerSchlauchsysteme für den Auto-Analyzer dienen. In manchen Publikationen. zu diesem Thema fehlen die elementarsten Angaben, so daß es oft unmöglich ist, danach $z u$ arbeiten. Diesem Zustand versucht ein Sonderausșchuß der „British Association of Clinical Biochemists" abzuhelfen, indem er in einer Arbeitsgemeinschaft die
Instrumente der "Technicon Company" geprüft hat und zu dem Schluß gekommen ist (1), daß jede neu veröffentlichte Methode, die mit dem Auto-Analyzer arbeitet, folgende Angaben enthalten muß:

a) Photographie einer Serie von Eichkurven aus der der Bereich und die Reproduzierbarkeit der Methode zu erkennen ist; b) Angabe der gemessenen Geschwindigkeit mit der der Probennehmer arbeitet; c) Angabe der gemessenen Geschwindigkeit des Schreibers; d) Angabe der wirklichen Umdrehungszahl der Walzenpumpe; e) Förderleistung der Pumpenschläuche bei 15 Sek./ Umdrehung der Walzenpumpe; f) Temperatur des Dialysators und anderer Heizbäder; g) Volumen der Heizschlangen, falls solche Verwendung finden; h) Raumtemperatur. 